\title{
The electron-gas pair density and its geminal description
}

\author{
P. Ziesche ${ }^{1}$ \\ Max Planck Institute for the Physics of Complex Systems, \\ Nöthnitzer Str. 38, D-01187 Dresden, Germany
}

Received XXX July, 2006; accepted in revised form YYY July, 2006

\begin{abstract}
Attempts to generalize the density functional theory are summarized. A possible pair density functional theory is linked to the Overhauser parametrization of the electrongas pair density. The importance of the cumulant partitioning is stressed and a modified Overhauser approach for the cumulant 2-body reduced density matrix, the contraction of which determines the 1-body reduced density matrix, is discussed.
\end{abstract}

Keywords: reduced density matrices, cumulant partitioning, generalized density functional theories, Kimball-Overhauser geminals

PACS: 71.10.Ca, 05.30.Fk, 71.15.Mb

The simplest quantum-kinematical quantity of a many-electron system (bound by $v_{\text {ext }}(\mathbf{r})$, described by $\hat{H}=\hat{T}+\hat{V}_{\text {ext }}+\hat{V}_{\text {int }}$, ground state) is its (1-body) density $\rho(1)$ with $1=(\mathbf{r}, \sigma)$. Density functional theory is an effective 1-body scheme, which provides the density $\rho(1)$ and the total energy $E$, supposed a certain density functional $E_{\mathrm{xc}}[\rho]$ is approximately known. But the 1-body reduced density matrix (1-matrix for short) $\gamma\left(1 \mid 1^{\prime}\right)$ and the pair density $\rho_{2}(1,2)$ remain unknown within this scheme. The 1-matrix $\gamma\left(1 \mid 1^{\prime}\right)$ contains not only the density with $\rho(1)=\gamma(1 \mid 1)$, but also the momentum distribution $n(k)$ (= diagonal of the Fourier transformed 1-matrix) and it enters the pair density in its cumulant partitioning $\rho_{2}(1,2)=\rho(1) \rho(2)-\gamma(1 \mid 2) \gamma(2 \mid 1)-u(1,2)$, where $u(1,2)$ is the diagonal of the cumulant 2-matrix $\chi\left(1\left|1^{\prime}, 2\right| 2^{\prime}\right)$. The corresponding partitioning of the interaction energy is $V_{\text {int }}=V_{\mathrm{H}}+V_{\mathrm{F}}+V_{\mathrm{C}}$ with $\mathrm{H}=$ Hartree, $\mathrm{F}=$ Fock, $\mathrm{C}=$ cumulant. The more general density-matrix functional theory may be considered as an effective 1-body scheme for $\gamma\left(1 \mid 1^{\prime}\right)$ and $E$, supposed $V_{\mathrm{C}}$ is approximately known as a 1-matrix functional $V_{\mathrm{C}}[\gamma]$. But the cumulant pair density remains unknown within this scheme. Pair-density functional theory [1]-[11] may be considered as an effective 2-body scheme for $\rho_{2}(1,2)$ and $E$, supposed $T$ is approximately known as a pair-density functional $T\left[\rho_{2}\right]$. But then the 1-matrix remains unknown.

It would be most desirable, if an effective 2-body scheme would be available for the cumulant geminals $\psi_{K}(1,2)$ and their occupancies $\nu_{K}$, such that $\chi\left(1\left|1^{\prime}, 2\right| 2^{\prime}\right)=\sum_{K} \psi_{K}(1,2) \nu_{K} \psi_{K}^{*}\left(1^{\prime}, 2^{\prime}\right)$ is the cumulant 2-matrix. Its diagonal gives the cumulant pair density $u(1,2)=\chi(1|1,2| 2)$ and from the contraction sum rule

$$
\int d 2 \chi\left(1\left|1^{\prime}, 2\right| 2\right)=\sum_{\kappa} \psi_{\kappa}(1) \nu_{\kappa}\left(1-\nu_{\kappa}\right) \psi_{\kappa}^{*}\left(1^{\prime}\right), \quad \gamma\left(1 \mid 1^{\prime}\right)=\sum_{\kappa} \psi_{\kappa}(1) \nu_{\kappa} \psi_{\kappa}^{*}\left(1^{\prime}\right)
$$

follows the 1-matrix by solving a quadratic equation. $\psi_{\kappa}(1)$ and $\nu_{\kappa}$ are the natural orbitals and their occupancies, respectively, which diagonalize the 1-matrix $\gamma\left(1 \mid 1^{\prime}\right)$. From $\rho(1)=\gamma(1 \mid 1)$ follow

\footnotetext{
${ }^{1}$ Corresponding author. E-mail: pz@pks.mpg.de
} 
$V_{\text {ext }}$ and $V_{\mathrm{H}}$, from $\gamma\left(1 \mid 1^{\prime}\right)$ follow $T$ and $V_{\mathrm{F}}$, and from $u(1,2)$ follows $V_{\mathrm{C}}: E=T+V_{\text {ext }}+V_{\mathrm{H}}+V_{\mathrm{F}}+V_{\mathrm{C}}$. Unfortunately almost nothing is known about the cumulant 2-matrix $\chi\left(1\left|1^{\prime}, 2\right| 2^{\prime}\right)$. In view of Eq. (1) it is sufficient to know the 3 -point function $\chi\left(1\left|1^{\prime}, 2\right| 2\right)$.

One way to learn something about these cumulant quantities may be to study them for the spin-unpolarized uniform or homogeneous electron gas (HEG)[12]. The advantage of this model is, that in its weak-correlation limit $r_{s} \rightarrow 0$, the cumulant 2-matrix $\chi\left(1\left|1^{\prime}, 2\right| 2^{\prime}\right)$ can be controlled through the well-known random-phase-approximation results for $n(k)$ and $\rho_{2}(1,2)$ [13]-[16]. There is still another motivation for such a study. Namely, on the one hand, there is the idea of Kimball and Overhauser [17]-[33], to parametrize the (dimensionless) HEG pair density as

$$
g(r)=2\left(\frac{1}{4} \sum_{L}^{+}+\frac{3}{4} \sum_{L}^{-}\right)\left\langle\mu(k) R_{l}^{2}(r, k)\right\rangle, \quad\langle\cdots\rangle=\int_{0}^{\infty} d(k)^{3} \cdots
$$

in terms of pair-density geminals $R_{l}(r, k)$ and corresponding weights $\mu(k)$. \pm stands for even, respectively, odd $l$, corresponding to the singlet, respectively, triplet components of $g(r)$. It turns out first a 2-body problem, which is easily treated separating-off the center-of-mass motion. It then remains a radial Schrödinger equation with an appropriately screened Coulomb repulsion and with scattering-state solutions $R_{l}(r, k)$. The geminal weight follows from $n(k)$ according to

$$
\mu(k)=\int_{0}^{\infty} d(K)^{3} n\left(\left|\frac{1}{2} \mathbf{K}+\mathbf{k}\right|\right) n\left(\left|\frac{1}{2} \mathbf{K}-\mathbf{k}\right|\right) .
$$

Notice the cumulant partitioning of the pair density as $g(r)=1-\frac{1}{2} f^{2}(r)-h(r)$ with $f(r)=$ Fourier transform of $n(k)$,

$$
f(r)=\int_{0}^{\infty} d(k)^{3} \frac{\sin k r}{k r} n(k), \quad \text { and } \quad 1-\frac{1}{2} f^{2}(r)=2\left(\frac{1}{4} \sum_{L}^{+}+\frac{3}{4} \sum_{L}^{-}\right)\left\langle\mu(k) j_{l}^{2}(k r)\right\rangle .
$$

Treating the electron-electron repulsion $\alpha r_{s} / q^{2}$ as perturbation, the cumulant pair density $h(r)$ is given by linked Feynman diagrams. The results of the Overhauser approach are promising, but on the other hand, there is the insight, that this approach violates the plasmon sum rule [33]. Is the mentioned search for a scheme, which provides the cumulant geminals with scattering states $\tilde{R}_{l}(r, k)$ and bound states $\tilde{R}_{n, l}(r)$ and - following from them - the cumulant pair density

$$
h(r)=2\left(\frac{1}{4} \sum_{L}^{+}+\frac{3}{4} \sum_{L}^{-}\right)\left(\left\langle\tilde{\mu}(k) \tilde{R}_{l}^{2}(r, k)\right\rangle+\sum_{n} \tilde{\mu}_{n} \tilde{R}_{n, l}^{2}(r)\right)
$$

a possible way out? $h(r)$ should have the long-range asymptotics in agreement with the plasmon sum rule and it has of course also to obey the cusp condition for $r \rightarrow 0$ [17].

\section{Acknowledgment}

The author wishes to thank P. Gori-Giorgi for valuable hints and P. Fulde for his support of this work. 


\section{References}

[1] P. Ziesche, Pair density functional theory - a generalized density functional theory, Phys. Lett. A 195, 213 (1994).

[2] P. Ziesche, Attempts Toward a Pair Density Functional Theory, Int. J. Quantum Chem. 30, 1361 (1996).

[3] M. Levy and P. Ziesche, The pair density functional of the kinetic energy and its simple scaling property, J. Chem. Physics 115, 9110 (2001).

[4] F. Furche, Towards a practical pair density-functional theory for many electron systems, Phys. Rev. A 70, 022514 (2004).

[5] P.W. Ayers, Generalized density functional theories using the $k$-electron densities: Development of kinetic energy functionals, J. Math. Phys. 46, 062107 (2005).

[6] P.W. Ayers and M. Levy, Generalized density-functional theory: Conquering the $N$ - representability problem with exact functionals for the electron pair density and the second-order reduced density matrix, J. Chem. Sciences 117, 507 (2005).

[7] P.W. Ayers and M. Levy, Using the Kohn-Sham formalism in pair density-functional theories, Chem. Phys. Lett. 415, 211 (2005).

[8] P.W. Ayers, S. Golden, and M. Levy, Generalizations of the Hohenberg-Kohn theorem: I. Legendre transform constructions of variational principles for density matrices and electron distribution functions, J. Chem. Phys. 124, 054101 (2006).

[9] P.W. Ayers and E.R. Davidson, Necessary conditions for the $N$-representability of pair distribution functions, Int. J. Quantum Chem. 106, 1487 (2006).

[10] P. Gori-Giorgi and A. Savin, Simple model for the spherically and system-averaged pairdensity: Results for two-electron atoms, Phys. Rev. A 71, 032513 (2005).

[11] P. Gori-Giorgi and A. Savin, System-adapted correlation energy density functional from effective pair interactions, Phil. Mag. 86, 2643 (2006).

[12] The spin-unpolarized HEG is characterized by the parameter $r_{s}$, from which follows the Fermi momentum as $k_{\mathrm{F}}=1 /\left(\alpha r_{s}\right), \alpha=(4 /(9 \pi))^{1 / 3}$ and the Fourier transformed Coulomb repulsion as $\alpha r_{s} / q^{2}$. Thus $r_{s}$ measures the interaction strength. It simultaneously measures the density, because a sphere with the radius $r_{s}$ (Wigner sphere) contains on average just one electron. But there is a non-vanishing probability of finding also 0 , as well as 2, 3 and more electrons in a Wigner sphere. Such particle number fluctuations in fragments have been studied by P. Ziesche, J. Tao, M. Seidl, and J.P. Perdew, How correlation suppresses density fluctuations in the uniform electron gas of 1, 2, or 3 dimensions, Int. J. Quantum Chem. 77, 819 (2000).

[13] E. Daniel and S.H. Vosko, Momentum Distribution of an Interacting Electron Gas, Phys. Rev. 120, 2041 (1960); I.O. Kulik, Zh. Éksp. Teor. Fiz. 40, 1343 (1961) [Sov. Phys. JETP 13, 946 (1961)].

[14] P. Gori-Giorgi and P. Ziesche, Momentum distribution of the uniform electron gas: improved parametrization and exact limits of the cumulant expansion, Phys. Rev. B 66, 235116 (2002).

[15] J.C. Kimball, High-density expansion of the electron-gas correlation function, Phys. Rev. B 14, 2371 (1976). 
[16] P. Ziesche and J. Cioslowski, The three-dimensional electron gas at the weak-correlation limit, Physica A 598 (2005).

[17] J.C. Kimball, Short-range Correlations and Electron-Gas Response Functions, Phys. Rev. A 7, 1648 (1973).

[18] A.W. Overhauser, Pair-correlation function of an electron gas, Can. J. Phys. 73, 683 (1995).

[19] P. Gori-Giorgi and J.P. Perdew, Short-range correlation in the uniform electron gas: Extended Overhauser model, Phys. Rev. B 64, 155102 (2001).

[20] M. Corona, P. Gori-Giorgi, and J.P. Perdew, Simple Physical Picture of the Overhauser Screened Electron-Electron Interactions, Phys. Rev. 69, 045108 (2004).

[21] B. Davoudi, M. Polini, R. Asgari, and M.P. Tosi, Self-consistent Overhauser model for the pair distribution function of an electron gas in dimensionalities $D=3$ and $D=2$, Phys. Rev. B 66, 075110 (2002).

[22] B. Davoudi, M. Polini, R. Asgari, and M.P. Tosi, Self-consistent theory of pair distribution functions and effective interactions in quantum Coulomb liquids, cond-mat/0206456.

[23] B. Davoudi, R. Asgari, M. Polini, and M.P. Tosi, Analytic theory of ground-state properties of a three-dimensional electron gas with arbitrary spin polarization, Phys. Rev. B 68, 155112 (2003).

[24] R. Asgari, M. Cardenas, M. Polini, B. Davoudi, and M.P. Tosi, Self-consistent Overhauser model for the pair distributiion function of an electron gas at finite temperature, condmat/0408293.

[25] I. Nagy, J.I. Juaristi, R. Díez Muiño, and P.M. Echenique, Short-range correlation in an electron gas: A scattering approach, Phys. Rev. B 67, 073102 (2003).

[26] I. Nagy, R. Díez Muiño, J.I. Juaristi, and P.M. Echenique, Spin-resolved pair-distribution functions in an electron gas: A scattering approach based on consistent potentials, Phys. Rev. B 69, 233105 (2004).

[27] R. Díez Muiño, I. Nagy, and P.M. Echenique, Calculation of pair correlation in a high-density electron gas: Constraints for effective interparticle potential, Phys. Rev. B 72, 075117 (2005).

[28] P. Ziesche, Cumulant 2-Matrix of the High-Density Electron Gas and the Density Matrix Functional Theory, Int. J. Quantum Chem. 90, 342 (2002).

[29] P. Ziesche and F. Tasnadi, Reduced density matrices, their spectral resolutions, and the Kimball-Overhauser approach, Ann. Phys. (Leipzig) 13, 2322002.

[30] P. Ziesche, K. Pernal, and F. Tasnadi, New sum rules relating the 1-body momentum distribution of the homogeneous electron gas to the Kimball-Overhauser 2-body wave functions (geminals) of its pair density, phys. stat. sol. (b) 239, 185 (2003).

[31] P. Ziesche, Normalization sum rules for the two-body scattering phase shifts of the electron-gas pair densities, Phys. Rev. B 67, 233102 (2003).

[32] P. Ziesche, The electron-gas pair density and its geminal representation. I. The geminal weight and its singularities, phys. stat. sol. (b) 241, 3544 (2004).

[33] P. Ziesche, The electron-gas pair density and its geminal representation. II. The long-range asymptotics of the Kimball-Overhauser geminals, phys. stat. sol. (b) 242, 2051 (2004). 